\title{
Analysis of Routing Protocols for Large Scale Multihop Multirate MANETS
}

\author{
Vincy Goyal ${ }^{1}$,Sunil Kumar Jangir ${ }^{2}$, Naveen Hemrajani ${ }^{3}$ \\ ${ }^{1}$ M.Tech*, Software Engineering, Suresh GyanVihar University Jaipur \\ ${ }^{2}$ Ph.D. Scholar, Department of Computer Science and Engineering,JECRC University Jaipur \\ ${ }^{3}$ Department of Computer Science and Engineering, Suresh GyanVihar University Jaipur \\ vincygoyal@gmail.com ${ }^{1}$, sunil.jangir07@gmail.com ${ }^{2}$, naven_h@yahoo.com³
}

\begin{abstract}
In this paper, we perform rigorous analysis of MANET routing protocols selected from different categories over various scenarios using a large set of performance evaluation metrics. The traffic that we model on source-destination pairs is the video streams that consist of varying sized data frames and the inter-packet time is very low. In this way, we can check the MANET routing protocols over varying data sets and can provide the analysis that among the existing MANET routing protocols which routing protocol is best suited for data transmission over MANETs. To analyze the behavior of various routing protocols during the data communication in MANETs, we generate simulation results over various MANET scenarios consists of varying number of nodes and source destination pairs. The simulation process is done by using the open source simulator NS-3. We generate and analyze the scenarios where the effects of data communication is evaluated and analyzed over the increase in network mobility and network data traffic. The work is helpful for the students working on the various issues on MANETs as attacks, Quality-of-
\end{abstract}

Service etc to identify which protocol they should use for their work as a base routing protocol.

\section{Council for Innovative Research}

Peer Review Research Publishing System

Journal: INTERNATIONAL JOURNAL OF COMPUTERS \& TECHNOLOGY

Vol 10, No 3 


\section{Introduction}

Efficient routing in mobile ad-hoc networks (MANETs) is a challenging task due to its varying physical channel characteristics, dynamic topology and un-centralized communication. Furthermore, multihop routing is required when the source-destination pairs are not in each other's communication range. Due to the above challenges these networks are considered unreliable for data communication and only provide best-effort services. The routing protocols used for route discovery in wired network are not used in wireless network due to their high signaling overhead caused by control messages. This signaling overhead consumes the scarce network bandwidth in wireless networks, increases interference and collisions and also drains the limited battery and other device resources.

Mobile ad-hoc network is a dynamic network formed by independent system of randomly moving mobile nodes. Nodes are connected through wireless links without utilizing the existing network infrastructure or any form of centralized administration. Each node is able to communicate directly with nodes in its transmission range. For nodes outside communication range, intermediate nodes are used to relay the message hop by hop. Hence, such networks are called "multi-hop" networks.

In an ad-hoc network, it is required that a node forwards or routes data packets on behalf of other nodes. Each node, therefore, acts as a host and a router, necessitating use of routing protocols to make routing decisions. Many routing protocols have been proposed by the researchers. The biggest challenge for routing protocols is to establish and reestablish routes in the face of dynamically varying network topology and network partitions due to node mobility. Depending on how the mobile nodes acquire and maintain routing information, MANET routing protocols can be classified as either reactive or proactive.

\section{Related Work}

In [1], authors present their evaluation results for the performance comparison of two reactive routing protocols i.e., AODV and DSR protocols. The authors compare the two protocols with increased network load and network mobility. The metrics on which the performance of these two routing protocols is evaluated is end-to-end delay, packet delivery ratio and routing overhead. The simulator used for the simulation is known as GLOBOSIM.

In [2], the authors present the performance evaluation of ADOV, DSR and DSDV routing protocols using the well known open source simulator known as ns-2. The metrics used for the performance measurement of the above three routing protocols are is end-to-end delay, packet delivery ratio, network throughput and routing overhead. The protocols are compared with change in packet size, inter-packet time and node mobility. In most of the scenarios their results shows that the AODV protocol outperforms the DSR and DSDV protocols.

Furthermore, in [3] the authors present the performance analysis of DYMO (modified and improved version of AODV protocol), AODV, OLSR and ZRP on various levels of mobility in the network. But the authors did not check the performance of the protocols in the presence of network load. Also the metrics used for the evaluation process are end-toend delay, packet delivery ratio, network throughput and routing overhead. All the simulations are performed on a network scalable simulator known as Qualnet. As their simulation in done on a more sophisticated simulator whose results are more closer to the physical testbad results it is considered a good work.

Finally, in [4] [5] [6] also authors from different countries present their performance evaluation for MANET routing protocols using free source or commercial simulators on a wide varieties of metrics and scenarios. As all the above work done on the evaluation of MANET routing protocols are either not evaluating the most popular routing protocols or they are not using all the required metrics to show their effectiveness or they are not using the all the possible available or presentable routing scenarios used by various MANET applications.

\section{SIMULATIONSAND PERFORMANCE EVALUATION}

We evaluate the performance of various MANET routing protocols over a wide range of network scenarios. The simulators used for generation of results are the Open source network simulator known as NS-3. The source-destination pairs in the network are created randomly and each value in the simulation graphs given below is the average of ten different simulation runs taken by changing the seed value of the network.

The simulation parameters and their values used for scenario creation and evaluation are given in Table 1. Some values like mobility related values, number of nodes in the network etc are given in a range because they are changes in every scenario.

Open source network simulator NS-3 is used to create simulation scenarios for development and analysis process of the proposed node-disjoint multipath routing protocol. To show the effectiveness and correctness of our proposed routing method we compare it with two the tradition reactive routing protocols known as on demand distance vector routing protocol and DSR

\section{A. Effect of increase in network load}

Figure 1 depicts the routing overhead of all the comparing routing protocols with increase in network load. As we can observe from the figure that the routing overhead of reactive protocols (i.e. AODV and DSR) is low initially and then it is increased with the network load. This is because as the number of source destination pairs increases in the network the routing overhead due to route 
discovery phase increases and it further increases with the increase in the route breaks caused by the increased traffic load in the network.

Table 1: Simulation Parameters

\begin{tabular}{|l|l|}
\hline Parameters & Values \\
\hline Simulator & NS-3 \\
\hline Simulation time & $600 \mathrm{sec}$ \\
\hline Network size & $1000 \times 1000$ sq. $\mathrm{m}$. \\
\hline $\begin{array}{l}\text { Number of } \\
\text { nodes }\end{array}$ & 60 \\
\hline $\begin{array}{l}\text { Transport } \\
\text { protocol }\end{array}$ & UDP \\
\hline $\begin{array}{l}\text { Routing } \\
\text { protocols }\end{array}$ & $\begin{array}{l}\text { AODV, DSR, OLSR and } \\
\text { ZRP }\end{array}$ \\
\hline Mobility model & Random way-point \\
\hline Pause time & 0 to $50 \mathrm{sec}$ \\
\hline Radio type & $802.11 \mathrm{~b}$ \\
\hline $\begin{array}{l}\text { Physical data } \\
\text { rate }\end{array}$ & $11 \mathrm{Mbps}$ \\
\hline Mobility speed & 0 to $30 \mathrm{~m} / \mathrm{s}$ \\
\hline Application & Constant bit rate (CBR) \\
\hline Frame size & 512 and 1000 bytes \\
\hline $\begin{array}{l}\text { Inter-packet } \\
\text { time }\end{array}$ & 33 ms \\
\hline
\end{tabular}

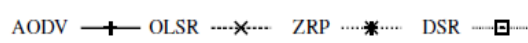

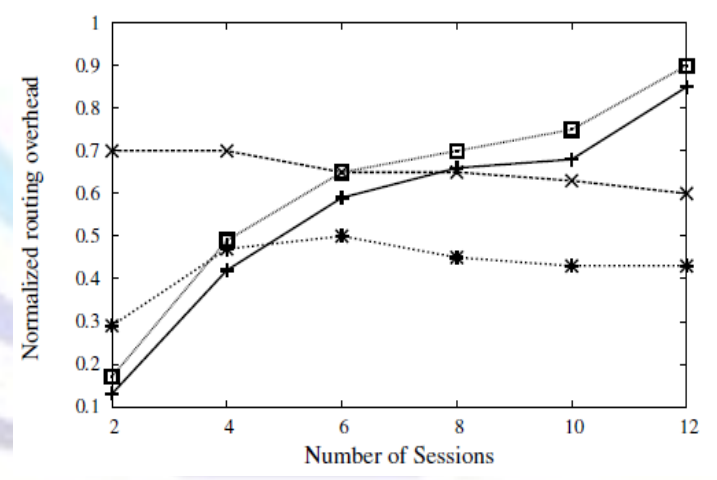

Figure 1 Overhead with increasing network load

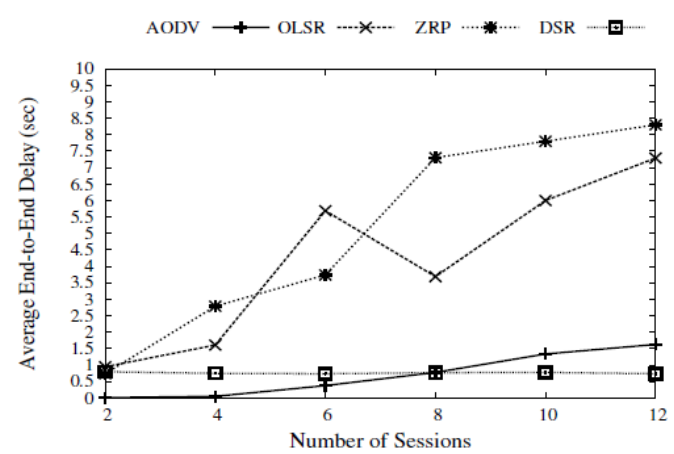

Figure 2 Average delay with increasing network load 
In Figure 2 we present the end-to-end delay (EED) of the four comparing routing protocols. As it can be seen from the Figure 2 that the EED of network increases with increase in the network load. This is because as the network load increases the data packets in an intermediate node's IP output queue also increases due to the congestion in the network. As the congestion increases the waiting time of data packets in the IP output queue before their fowarding towards their destinations also increases.

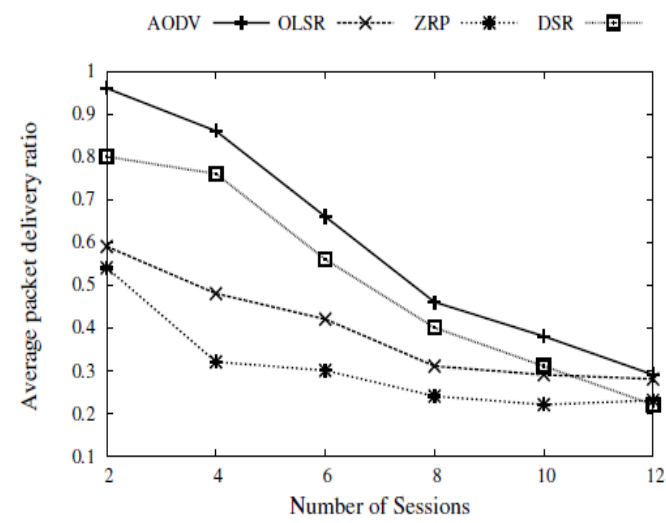

Figure 3 Packet delivery ratio with increasing network load

In Figure 3 and Figure 4, we can see the packet delivery ratio (PDR) and network throughput of all the four comparing routing protocols with increase in number of data sessions in the network. As we can observe from Figure 3 that the both PDR and network throughput of all the four routing protocols is higher when the data load in the network is lower than its total bandwidth it creates congested routes for the routing process. Therefore, increases the PDR and network throughput of reactive routing protocols.

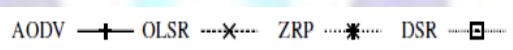

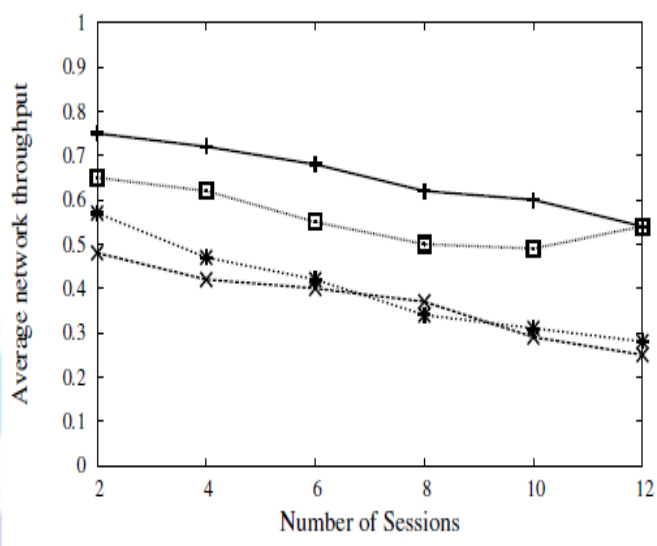

Figure 4 Network throughput with increasing network load

As the load in the network increases the number of packet losses due to collisions and interference caused by nearby flows increases. Also we can see from Figure 3 that AODV and DSR routing protocols are well suited for moderate load networks as compared to the OLSR and ZRP protocols as their PDR and network throughput is higher in small and moderate sized networks as compared to OLSR and ZRP. This is due to the reactive route discovery process of the AODV and DSR which avoids the routes that are more congested and discovers the less

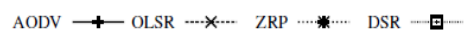

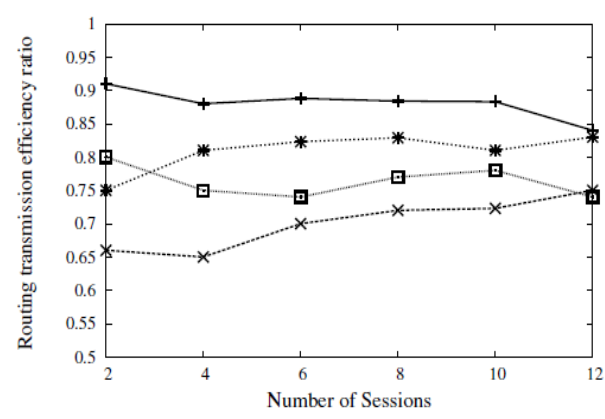

Figure 5 Routing transmission efficiency with increasing network load 
The routing transmission efficiency (RTE) of the four comparing routing protocols in this thesis is shown in Figure 5 . As it can be seen from the Figure 5 that RTE of the AODv is highest and remain constant with the increase in the network load and the RTE of the OLSR is lowest at the start and then increases slightly as the netwrok load increases. This is because in AODV the route discovery phase will avoid the congested routes during the route dicovery process and carefully select the route which is less congested therefore the number of packets that are tranmsitted and also acknowleged are more in AODV then other routing protocols. On the other hand, the RTE is constant with the increase in network load because as the network load increases the number of packets that are trnamsitted in the network are not increases significantly. This is because the packets are dropped due to no route or due to overflow of the IP output queue. So less packets are transmitted therefore less packets are collided and due to this the RTE remains constant with the increase of the network load.

\section{Conclusions and Future Directions}

In this thesis, we have presented the detailed and regrous performance evaluation of mobile ad-hoc network routing protocols. The evaluation and performance comparision is done on various scenarios in which network load and mobility in the network changes constantly. The mertics used for the analysis of the routing protocols are selected in such a way so that they can cover all the possible behavious of the routing protocols and provide their uses on different kinds of sceanrios. We uses five evaluation metrices that are as follows: end-to-end delay, packet delivery ratio, network throughput, routing transmission efficiency and routing overhead.

The simulation results shown in the Chapter 4 shows that reactive routing protocols are more suitable in most of the scenarios and situation in the MANETs as compared to the proactive and hybrid routing protocols. The results also shows that the AODV routing protocols is the best suited routing protocols for MANETs as it is able to adapt its route discovery phase according to the dynamic conditions of the wireless network. Furthermore it has been observed from the simulation results that the routing protocols given for the MANET routing are only best-effort routing protocols i.e., they can only provide support for a data communication on the best-effort way. Therefore, the routing protocols of MANETs are not able to deliver data correctly when the network load increases and mobility of network changes very frequently.

Due to the best-effort nature of the routing protocols of the MANETs. In the future we will try to enhance their functionalities so that they can able to work in large networks with given constraints and also able to provide the service which may be of application specific.

\section{References}

1Chaba, Y.; Singh, Y.; Joon, M., "Simulation Based Performance Analysis of On-Demand Routing Protocols in MANETs," Computer Modeling and Simulation, 2010. ICCMS '10. Second International Conference on, vol.3, no., pp.80,83, 22-24 Jan. 2010

2Tuteja, A.; Gujral, R.; Thalia, S., "Comparative Performance Analysis of DSDV, AODV and DSR Routing Protocols in MANET Using NS2," Advances in Computer Engineering (ACE), 2010 International Conference on , vol., no., pp.330,333, 20-21 June 2010

3Rahman, M.A.; Anwar, F.; Naeem, J.; Abedin, M.S.M., "A simulation based performance comparison of routing protocol on Mobile Ad-hoc Network (proactive, reactive and hybrid)," Computer and Communication Engineering (ICCCE), 2010 International Conference on , vol., no., pp.1,5, 11-12 May 2010

4Maan, F.; Mazhar, N., "MANET routing protocols vs mobility models: A performance evaluation," Ubiquitous and Future Networks (ICUFN), 2011 Third International Conference on , vol., no., pp.179,184, 15-17 June 2011

5Vir, D.; Agarwal, S.K.; Imam, S.A., "Quantitative analyses and evaluation of MANET routing protocols in effect of varying mobility model using Qual-Net simulator," Information and Communication Technologies (WICT), 2012 World Congress on , vol., no., pp.915,921, Oct. 30 2012-Nov. 22012

6Shobana, M.; Karthik, S., "A performance analysis and comparison of various routing protocols in MANET," Pattern Recognition, Informatics and Medical Engineering (PRIME), 2013 International Conference on , vol., no., pp.391,393, 2122 Feb. 2013. 